\title{
HRCT Surrogate for Vaccine Efficacy?
}

\author{
Anirudh Kohli ${ }^{1}$ \\ ${ }^{1} \mathrm{HOD}$, Department of Imaging, Breach Candy Hospital, Mumbai, \\ India
}

Indian J Radiol Imaging 2021;31:243-244.

The Present Pandemic Continues Unabated with Increasing Ferocity with Each Wave. The only way out of this pandemic appears to be creating herd immunity to the virus, either innate from infection or vaccination. There are three important terms to determine the efficacy of vaccines, namely vaccine efficacy, vaccine effectiveness, and breakthrough infections. Vaccine efficacy refers to the ability of the vaccine to reduce the incidence of infection. In a controlled clinical environment, the number of vaccinated individuals getting infected versus the number getting infected after receiving a placebo. ${ }^{1}$ Vaccine effectiveness refers to the rate of infection occurring in the real world. More importantly is the term breakthrough infections, when infection breaks through the vaccine shield. Breakthrough infections have been seen with all vaccines as the efficacy and effectiveness of all vaccines are not $100 \%$. In view of breakthrough infections, the real effectiveness of vaccines will be to reduce the severity of infection in terms of the development of pneumonia, hospitalization, and mortality. Merely adding up the number of positive RT PCR following vaccination will be not of much value; however, what would be important is a measure of the severity of breakthrough infections as compared with non-vaccinated individuals or between different vaccines/vaccination schedules/vaccine combinations.

Computed tomography (CT) has played a stellar role in this pandemic, much against the recommendations and guidelines issued by numerous national societies. ${ }^{2}$ CT has worked as a public service tool in detecting false-negative RT PCR cases, thus aiding in the early isolation of super spreaders. $^{3}$ CT has also assisted in clinical management by demonstrating lung involvement and complications of COVID-19. Probably, the most important contribution of CT has been the CT severity index or percentage of lung involvement. Not only does the CT severity index demonstrate the extent of lung
Address for correspondence Anirudh Kohli, MD, DNB, DMRD, HOD, Department of Imaging, Breach Candy Hospital, Mumbai 400026, India (e-mail: dranirudhkohli@gmail.com).

involvement, which helps prognosticate, but has played a pivotal role in triaging, reducing the burden on medical facilities. The severity of lung involvement correlates extremely well with oxygen requirement, type of oxygen requirement invasive/non-invasive, ICU admissions. ${ }^{4}$ Because the CT severity index is a surrogate for determining the severity of illness, it would be a useful and realistic tool to evaluate the real effectiveness of vaccines in reducing the severity of the disease. Initial studies applying this thought process have been performed, the initial results have demonstrated a reduced CT severity index in vaccinated individuals as compared with non-vaccinated individuals. ${ }^{5}$

These results would be important to understand the value and contribution of vaccination, especially in individuals who are not keen on vaccination thus propelling the planet toward herd immunity.

\section{References}

1 eRef) Vaccine efficacy, effectiveness and protection. Available at: https://www.who.int/news-room/feature-stories/detail/ vaccine-efficacy-effectiveness-and-protection. Accessed July 14, 2021eRef

2 A. COVID-19: the second wave-are there lessons from the first wave to prepare us for the second wave? Indian J Radiol Imaging 2021;31(1):1-2

3 Kohli A. Was non-utilisation of computed tomography as a public health tool a costly lapse in closing the pandemic? Indian J Radiol Imaging 2021;31(1(Suppl 1):S1-S3

4 Kohli A, Jha T, Pazhayattil AB. The value of AI based CT severity scoring system in triage of patients with Covid-19 pneumonia as regards oxygen requirement and place of admission. Indian J Radiol Imaging 2021;31(1):S61-S69

5 Madhu P, Santhosh D, Kiran M. Comparison study of lung involvement in vaccinated and un vaccinated Covid patients. International Journal of Health and Clinical Research $2021 ; 4(10): 229-233$
DOI https://doi.org/ $10.1055 / \mathrm{s}-0041-1736127$ ISSN 0971-3026 (c) 2021. Indian Radiological Association.

This is an open access article published by Thieme under the terms of the Creative Commons Attribution-NonDerivative-NonCommercial-License, permitting copying and reproduction so long as the original work is given appropriate credit. Contents may not be used for commercial purposes, or adapted, remixed, transformed or built upon. (https://creativecommons.org/licenses/by-nc-nd/4.0/).

Thieme Medical and Scientific Publishers Private Ltd. A-12, Second Floor, Sector -2, NOIDA -201301, India 
